\title{
Virtual Prototyping and Real-Time Simulation of Heavy Equipment Manipulators including Elastic Deformations and Hydraulics
}

\author{
D. Schramm, D. Franitza, and W. Lalo
}

\begin{abstract}
The efficient and unerring development of sophisticated manipulation machinery strongly relies on the efficient use of CAE (Computer Aided Engineering) methods. Meanwhile these methods more and more spread into areas, which have been so far domains of so called VR (Virtual Reality). This encompasses the design aspect as well as the simulation of an already defined device under given workload and working environment along with the option for an easy adaptation to any change of the requirements.
\end{abstract}

Whereas the efficient and reliable simulation of the real world behaviour of rigid body models is state of the art even for complex machines the inclusion of elastic effects especially in real time simulations is still the cutting edge of technical simulation. This includes the elasticity of the multibody structure itself as well as the influence of compliant hydraulic subsystems coupled to the deformable structure.

The paper looks at the off-line as well as at the real-time simulation of elastic hydraulically driven heavy multi-axes manipulators like e.g. concrete pumps. The application of the tools to support the development of heavy machines will be discussed. Along with the simulation of the operation behaviour the work done also focuses on the support of the advanced man-machine-interfaces used to operate the device.

Various modules have been developed and proved to easily include hydraulic components like interfaces to Hydraulic Simulation Software Systems, like e.g. DSHplus into the description of the dynamic behaviour of the basic elastic mechanical structure.

There is also a strong need to run simulation programs for the devices under consideration in real-time mode. Therefore strategies were developed and applied to various examples to simplify the respective model architectures along with the simulation algorithms in order to allow real-time simulations reflecting the actual properties of the device with still a high precision level. The models created are suited to be converted into a 'real-time code' with the option to be uploaded onto the microcontroller of the machine hardware.

The application of the methods presented will be explained at an example based on real world equipment including on-line animation.

D. Schramm is head of the Chair of Mechatronics, Department of Mechanical Engineering, University of Duisburg-Essen, D-47057 Duisburg, Germany (e-mail: schramm@mechatronik.uni-duisburg.de).

D. Franitza is chief engineer at the Chair of Mechatronics, Department of Mechanical Engineering, University of Duisburg-Essen, D-47057 Duisburg, Germany (e-mail: franitza@mechatronik.uni-duisburg.de).

W. Lalo is with the Chair of Mechatronics, Department of Mechanical Engineering, University of Duisburg-Essen, D-47057 Duisburg, Germany (email: lalo@mechatronik.uni-duisburg.de).
Keywords-CAE, Real-time simulation, multibody systems.

\section{INTRODUCTION}

Heavy Equipment Manipulators typically are based on mechanisms fulfilling extreme mechanical properties, hydraulic actuators and complex motion control systems. Moreover depending on the actual design of the manipulator arms the elastic behaviour of the system components cannot be totally neglected. The design and simulation of this type of system especially under real time conditions is still a challenge and requires efficient methods to set up and solve the equations of motion.

A very efficient approach is the application of the concept of transmission elements. These transmission elements are used in the first chapter of this article to describe the properties and the dynamics of the mechanical as well as the hydraulic sub-system in a compatible manner.

As the off-line simulation even of this type of complex hybrid system is not really an obstacle during the design phase of these manipulators, real-time as well as hardware-in-the-loop simulations are still a challenge. The root causes are limitations of the computer hardware used along with bottlenecks caused by the bus architecture of the system. The article addresses methods to solve these problems and explains their application on selected examples.

\section{Mechatronic Modelling}

\section{A. The Concept of Transmission Elements}

1) Mechanical Modelling

Considering the modelling of complex mechanical multibody systems (MBS) a description in minimal coordinates is a suitable way to generate the equations of motion in a compact and efficient manner [1] according to

$$
\mathbf{M}(\mathbf{q}) \ddot{\mathbf{q}}+\mathbf{b}(\mathbf{q}, \dot{\mathbf{q}})=\mathbf{Q}(\mathbf{q}, \dot{\mathbf{q}})
$$

Thereby we define 
$\mathbf{M} \in \mathfrak{R}^{f \times f}:$ Generalized mass matrix, which is symmetric and positive definite,

$\mathbf{b} \in \mathfrak{R}^{f}: \quad$ Generalized Coriolis, gyroscopic and centrifugal force vector,

$\mathbf{Q} \in \mathfrak{R}^{f}: \quad$ Generalized applied force vector.

The key idea of handling closed kinematical loops is to exploit the method of characteristic pairs of joints to state six constrained equations of an individual multibody loop in wherever possible closed form and the concept of transmission elements (Fig. 1)

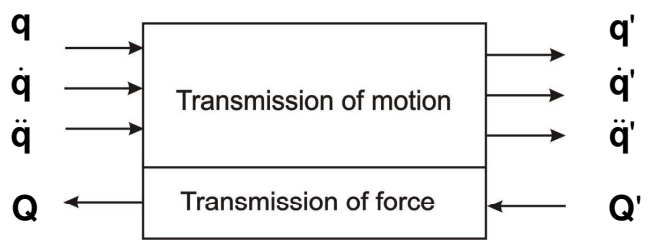

Figure 1: Kinetostatic transmission element

Thereby the transmission of motion is defined by the transmission of the position

$$
q^{\prime}=\varphi(q)
$$

and its time derivatives, i.e. velocity

$$
\dot{\mathrm{q}}^{\prime}=\mathrm{J}_{\varphi} \dot{\mathrm{q}}
$$

and acceleration

$$
\dot{q}^{\prime}=J_{\varphi} \ddot{q}+\dot{J}_{\varphi} \dot{q}
$$

where the Jacobian $\mathbf{J}_{\boldsymbol{\varphi}}$ is determined by

$$
\mathbf{J}_{\varphi}=\frac{\partial \varphi}{\partial \mathbf{q}} \in \Re^{m \times n}
$$

At last the transmission of force is obtained by

$$
\mathbf{Q}=\mathbf{J}_{\varphi}^{\mathbf{T}} \mathbf{Q}^{\prime}
$$

For modelling the multibody kinematics and dynamics in a very efficient manner the software MOBILE [1] has been developed and established. It is based on the object-oriented programming language $\mathrm{C}++$ and thus enables an operating-system independent application. This is beneficial for the use of the generated MOBILE models in real-time system software to provide modelbased control. Additionally several interfaces enable to connect the MBS to external software environments, which root in a different field of engineering, i. E. hydraulic subsystems.

The mechanical structure of heavy equipment can be repre- sented by a multibody system consisting of several joints and rigid or elastic links as well as coupled kinematical loops. The complexity of the mechanical subsystem may vary from case to case. The examples range from a simple pendulum to large scale walking machines $[3,4]$ with several hydraulically actuated geometrically nonlinear closed loops.

\section{2) Elastic Multibody Systems}

For large scale manipulators elastic deformations can rarely be neglected due to self-weight or dynamic excitation. Therefore so-called elastic coordinates have been implemented into MOBILE to map linear-elastic deflections onto a semi-rigid structure [5]. Along a one-dimensional structure the generalized coordinates $\mathbf{q}$ at a given point can be composed of rigid and elastic shares according to

$$
\mathbf{q}(\mathrm{Q})=\text { qrigid }+ \text { qelast }(Q) \text {. }
$$

Although small values for local strain are expected the accumulation of the elastic deflection over a long manipulator arm leads to a visible shift of the end-effector as to be seen in Fig. 7. Having modelled elasticity of multibody structures it is possible to predict a deformed behaviour of the overall structure, which can be considerably different from a strictly rigid framework.

\section{3) Hydraulic Modelling}

The state variables in hydraulic subsystems are considered to be pressure variables at certain nodes within the hydraulic circuit. The pressure development can be described using the pressure build-up equation [6], considering the pressure depending hydraulic capacity as well as the equilibrium of volume flow variables through the respective node. Tab. 1 summarizes the analogy of the mechanical and hydraulic transmission elements.

\begin{tabular}{cc}
\multicolumn{2}{c}{ TABLE 1: ANALOGY OF TRANSMISSION ELEMENTS } \\
\hline Mechanics & Hydraulics \\
\hline Reference System & Pressure Node \\
Displacement & Pressure \\
Force & Volume Flow \\
\hline
\end{tabular}

\section{4) Overall Modelling}

Due to the concept of creating several transmission elements which map a set of input variables onto its set of output variables the coupling of naturally different subsystems can be done by virtually connecting the respective output signals to the appropriate input variables. Every connection of transmission elements consists of two sets of variables, one of which is called "through" variables and the others "across" variables (Fig. 2).

"Across" variables usually are the same for all connected elements, except for explicit external loads (in mechanical sense: kinematical variables), while "through" variables form the appropriate equilibrium (like kinetic variables in mechanic respect). Fig. 3 describes the interdependence of mechanical and hydraulic subsystems. 


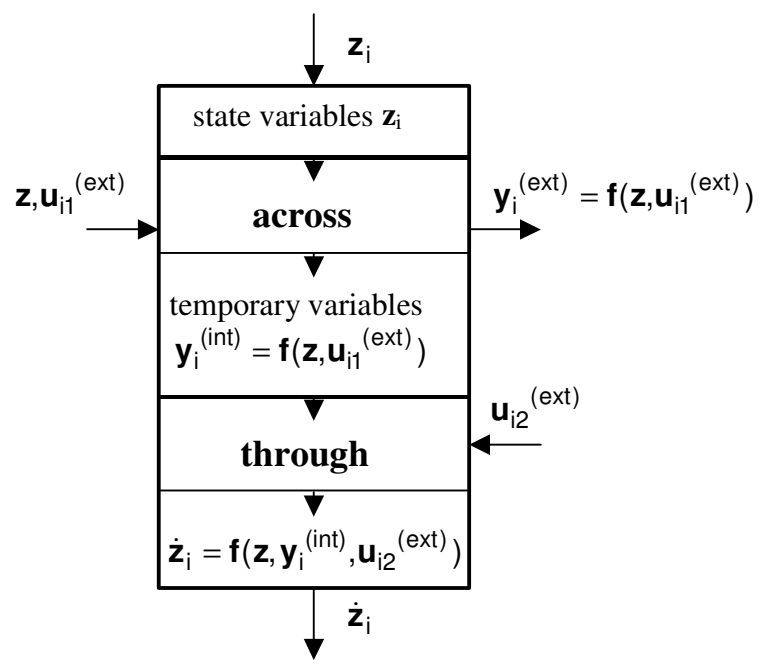

Figure 2: Interface of subsystems

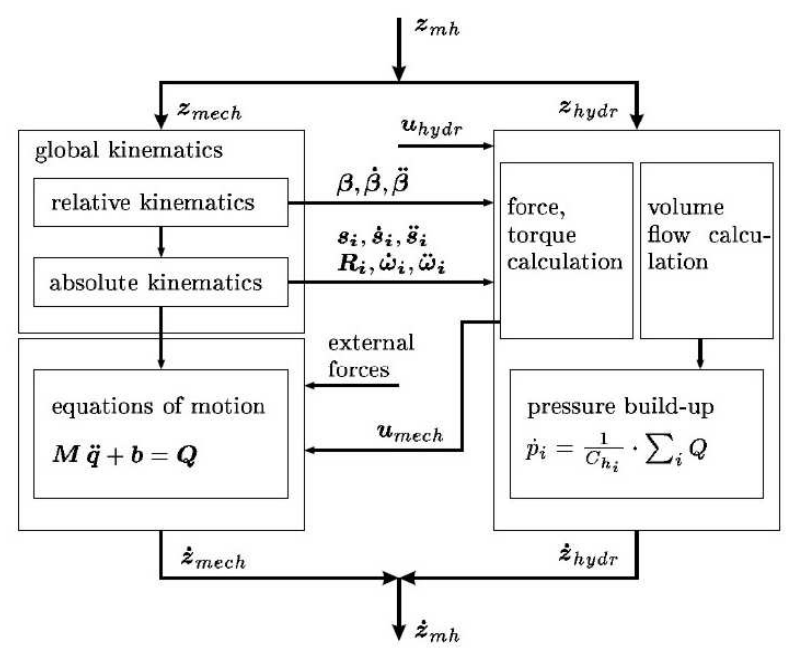

Figure 3: Coupling of mechanics and hydraulics

\section{MOdELBASED CONTROL AND SIMULATION}

With the complete system represented by a model a modelbased controller can be set up. While the real system works basically the same way the model does, various enhancements can be implemented.

For instance it is possible, using a sufficiently fast simulation of the real system running in the background, to estimate in advance the future reaction of the system due to time extrapolation of the current state. As the change of all system variables is known at a given time, estimation to a slightly advanced time can be given. This way it is possible not only to react to a situation but also to correct the sources for control errors.

\section{A. Real-Time Approach}

For purposes of machinery control certain safety requirements must be met. One of those is the need for a controllability, which mainly relies on a scheduler, controlling the flow of information inside the controlled system. In most cases a realtime system is set up to ensure the demand of regular controller tasks, consisting of reading available sensor data and sending necessary actuator intervention.

A regularly observed bottleneck appears to be the bus architecture. As the CAN protocol has become more and more industrial standard. Meantime also drivers for real-time systems are or become available. In the past this missing drivers caused lots of problems in this sort of applications.

\section{B. Hardware-In-The-Loop}

If all requirements for modelbased control are met, the simulation is ready to be connected to a single part out of the real overall structure. This approach is already state of the art in automotive industry, as controller boxes are connected to so called Hardware-In-The-Loop environments [7]. They consist of a simulation, in this specific case of a vehicle dynamics simulation of a given car, and a connection to the test rig or the device to be tested, commonly via CAN-connector (Controller Area Network, Fig. 4).

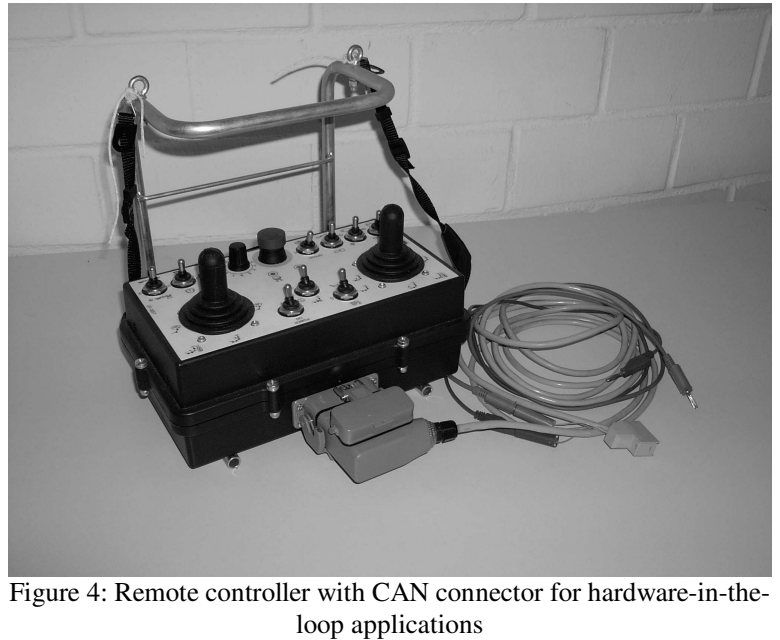

The real device cannot distinguish between reality and simulation. All it does is to control the variables it is designed for.

\section{EXAMPLES}

\section{A. Mobile Concrete Pumps}

Mobile concrete pumps belong to the heavy equipment manipulators and are typical mechatronic systems. In service a built-in pump delivers the liquid concrete through inlet pipes along a boom package and finally pours it out by a flexible tube.

With respect to kinematics the boom package is a redundant manipulator. It may consist of up to five extension arms connected one by one by horizontally parallel-arranged rotary joints and thus represents a planar manipulator. Each boom is 
driven by a linear hydraulic actuator arranged within a four-bar linkage. In this manner closed kinematical loops arise which have to be peculiarly treated. The base is connected to a rotary joint with a vertical rotation axis, which allows the spatial motion of the end-effector (Fig. 5).

The complete boom package is mounted on a tractor in order to enable a mobile service.

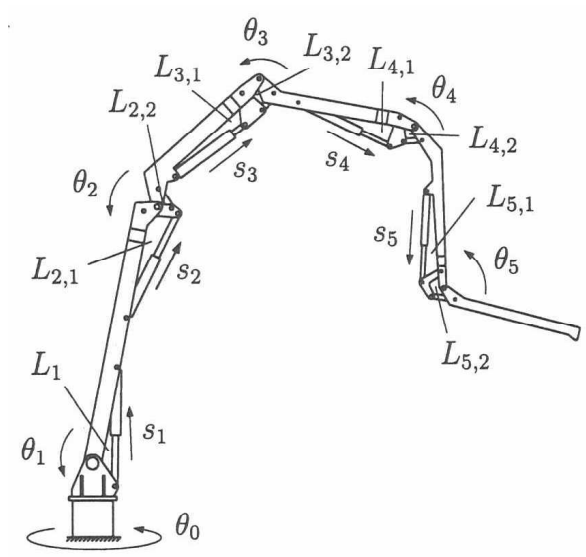

Figure 5: Manipulator with local closed kinematical loops

The outstretched extension arms may reach a length of about $60 \mathrm{~m}$. Due to these dimensions and the emerging vibrations caused by the concrete pump during service the vehicle has to be supported sufficiently. As a rule the support is realized by two struts at each side of the truck, which can be extended and lowered (Fig. 6).

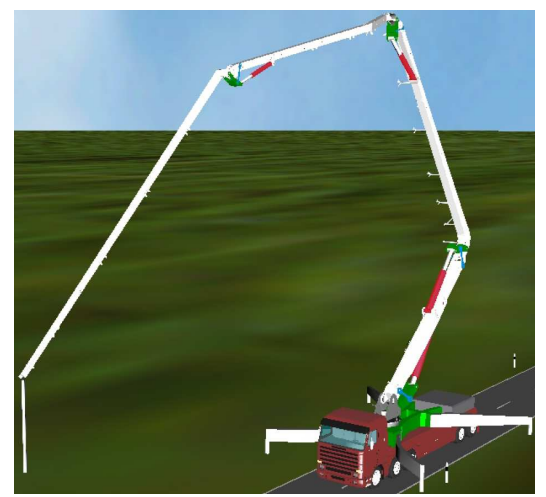

Figure 6: virtual truck-mounted concrete pump in service

\section{1) Modelling}

As for this kind of problem the kinematical transmission of the positions of the hydraulic cylinders, which represent the input of the system, to the position and orientation of the endeffector or tool centre point (TCP) is one essential subtask that has to be solved. As a rule this transmission of motion is a nonlinear problem consisting of one or more kinematical loops. These are supposed to be solved in a closed form in order to provide a fast, i.e. real-time calculation.

This problem is a typical challenge for the fields of mecha- tronics. As explained above a modelbased control of the system can be established. For this purpose a virtual model of the real system consisting of a mechanical and hydraulic subsystem is set up.

In dependence of the construction of each extension arm elastic deformations occur which cannot be neglected. This characteristic has large-scale consequences considering the accurate positioning of the end-effector, which can oscillate up to half a meter and more. Additionally the compressibility of the hydraulic oil may have to be regarded as it also causes further oscillations.

The following simulation result taken from [5] (Fig. 7, Fig. 8) shows this influence by means of a static deflection. Initially all extension arms are horizontally stretched according to the starting position (0) without any elastic deformations and closed valves.

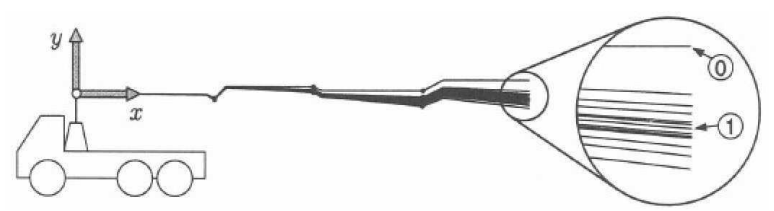

Figure 7: Static deflection

Influenced by its dead weight and a payload of $150 \mathrm{~kg}$ at the tool centre point (TCP) the system is let off. According to Fig. 8 the system reaches the static deflection after $12 \mathrm{~s}$ at point (1).

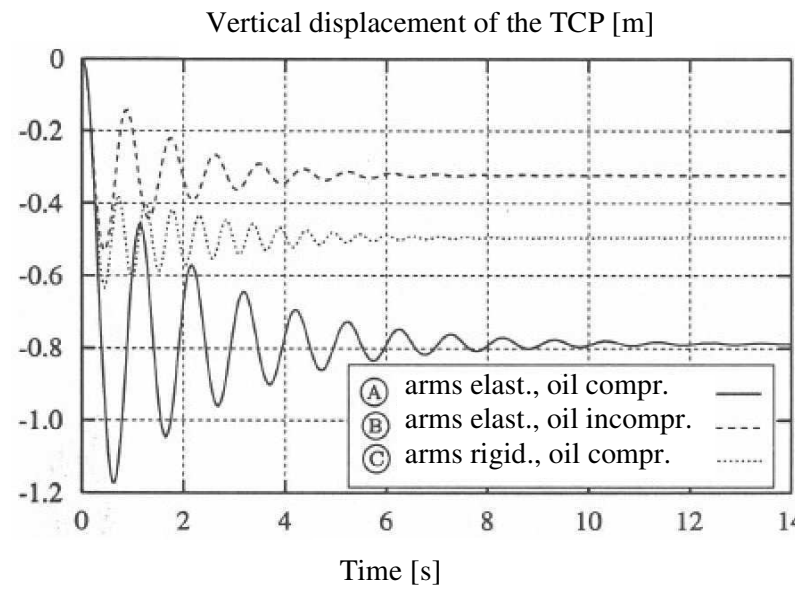

Figure 8: vertical displacement of the TCP considering dead weight and payload.

\section{2) Control}

The Simulation of a concreting manoeuvre shows a typical task of how to decrease the unwanted motion of the endeffector. With the help of the modelbased control the oscillations can be reduced to a minimum.

Fig. 9 shows both the controlled and uncontrolled motion of 
a typical concreting service. Hereby the end-effector is defined to place the concrete along a given trajectory from the initial point $\mathrm{A}$ to the end point $\mathrm{B}$.

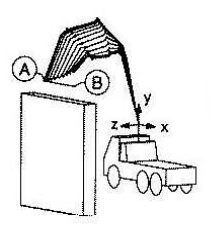

uncontrolled

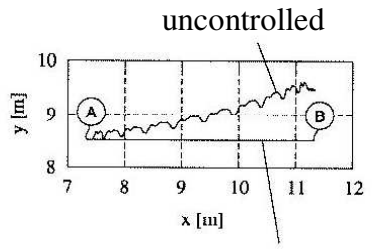

controlled

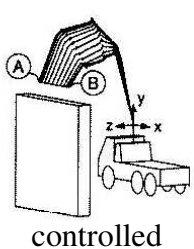

controlled
Figure 9: Simulation of a concreting manoeuvre

The uncontrolled motion removes itself from the given trajectory and additionally oscillates in vertical direction. In contrast the controlled motion of the end-effector and the given trajectory are nearly identical (Fig 10).

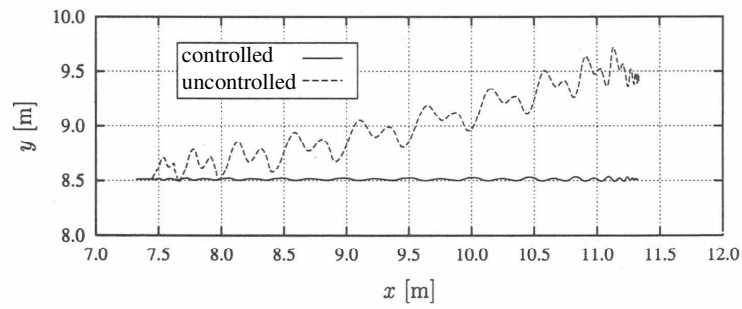

Figure 10: Trajectory of the end-effector of the elastically modelled concrete pump during concreting

\section{3) Collision Avoidance}

Typical of these kinds of manipulators is their redundancy. It allows certain flexibility with respect to obstacles, which may be located within the workspace of the manipulator. As the current configuration of the extension arms are known, an obstacle avoidance algorithm can be established by the help of the theory of the elastic band [8] provided that the current location of the obstacles is known (Fig 11). Thus the obstacle can also be a moving object, e.g. an incoming excavator.

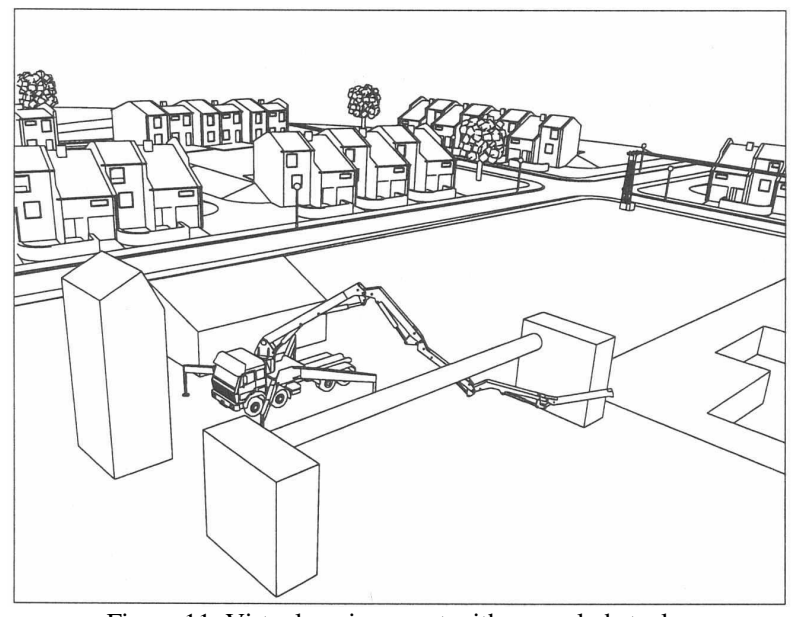

Figure 11: Virtual environment with several obstacles

The idea of this concept is to deflect the trajectory to be traced with the help of spring potentials. These potentials are defined to cover the obstacle with a certain safety distance and deform the trajectory if it enters the safety proximity.

Fig. 12 shows typical obstacles, which are covered by their corresponding potential fields using the safety distance $\mathrm{d}_{0}$.
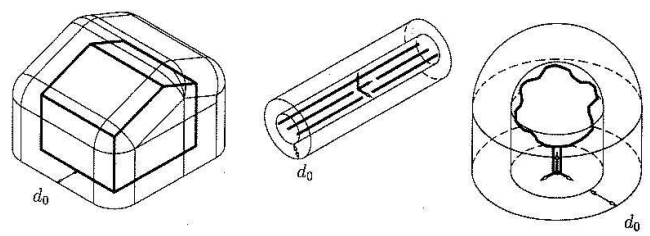

Figure 12: Potential fields of some obstacles

Fig. 13 shows an example of collision avoidance. The task is to trace a segment of a circle between the points $\mathrm{A}$ and $\mathrm{B}$. The consideration of the tower to be an obstacle generates a motion according to Fig. 13.b by defining a potential field around the tower. Otherwise a collision with the tower occurs (Fig. 13.a).

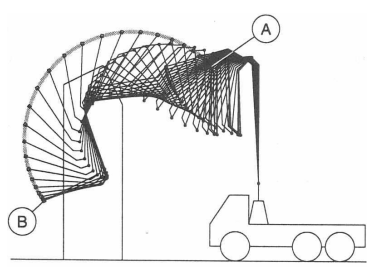

a) without collision avoidance

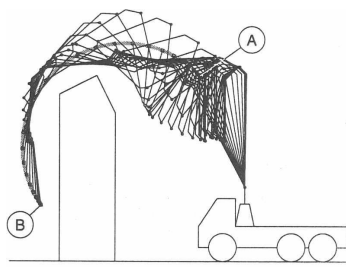

b) with collision avoidance
Figure 13: Circumscribing a tower

\section{4) Hardware-In-The-Loop}

The existence of a virtual model allows the Hardware-inthe-Loop Simulation. For instance a remote controller can be connected to the computer and thus to the virtual model. Thereby the application of the interface device, e.g. CANCommunication, which is used for the real system, can be directly adopted by the computer and therefore tested with the help of the virtual model.

The communication between virtual model and real system components can also be extended to check and study the behaviour of sensors to be applied for the real system. Using standard communication protocols enables an a priori control of these devices to test and establish appropriate algorithms with the help of the virtual model before testing them with the real system.

\section{CONCLUSION}

The concept of multibody simulation along with the use of transmission elements in order to describe the mechanical as well as the hydraulic behaviour is used to simulate the dynamic behaviour of heavy equipment manipulators including elastic effects of the manipulator arms. The description in minimal 
coordinates is used to generate the equations of motion for the complete system in a very efficient way.

Due to the generation of the virtual model a modelbased control can be established. Simulation results of a typical concreting manoeuvre show that by the help of modelbased control the elastic effects of the manipulator arms can be reduced to a minimum.

The application to problems where obstacles are located in the workspace of the manipulator is shown using an obstacle avoidance algorithm based on the theory of the elastic band.

\section{REFERENCES}

[1] A. Kecskeméthy, M. Hiller, "An object-oriented approach for an effective formulation of multibody dynamics," Computer methods in Applied Mechanics and Engineering, vol. 115: pp. 287-314, 1994.

[2] A. Kecskeméthy, "An object-oriented tool-set for the efficient modeling of mechatronic systems," in Hiller, M. and Fink, B. (editors), Proceedings of the Second Conference on Mechatronics and Robotics, pp. 447462, Duisburg/Moers, Germany, 1993.

[3] J. Müller, M. Hiller, ,Design of an energy optimal hydraulic concept for large scale combined legged and wheeled vehicle ALDURO”, in Proceedings of the $10^{\text {th }}$ IFToMM World Congress, Oulu, Finland, 1999

[4] J. Müller. (1999). "Simulation hydraulischer Systeme in MOBILE und DSHplus," Institutsbericht IB98-3, Fachgebiet Mechatronik, Universität Duisburg-Essen, 1998.

[5] M. Schneider, M. Hiller, "Modeling, simulation and control of flexible manipulators," European Journal of Mechanics, A/Solids, vol. 16, special issue: pp. 127-150, 1997.

[6] W. Backé, H. Murrenhoff, "Grundlagen der Ölhydraulik," Umdruck zur Vorlesung, Institut für fluidtechnische Antriebe und Steuerungen, RWTH Aachen, 10. Auflage, 1994.

[7] M. Torlo, T. Bertram, „Development of Fault-tolerant Distributed Vehicle Systems", VDI Würzburg, 2000.

[8] O. Khatib, "Real-time obstacle avoidance for manipulators and mobile robots", The International Journal of Robotics Research, vol. 5: pp. 9098, 1986. 